\title{
Memory for sequences of events impaired in typical aging
}

\author{
Timothy A. Allen, ${ }^{1,3}$ Andrea M. Morris, ${ }^{1,2,3}$ Shauna M. Stark, ${ }^{1}$ Norbert J. Fortin, ${ }^{1}$ \\ and Craig E.L. Stark ${ }^{1}$ \\ ${ }^{1}$ Center for the Neurobiology of Learning and Memory and Department of Neurobiology and Behavior, University \\ of California, Irvine, California 92697-3800, USA; ${ }^{2}$ Department of Health Policy and Management, University of \\ California, Los Angeles, California 90095-1772, USA
}

\begin{abstract}
Typical aging is associated with diminished episodic memory performance. To improve our understanding of the fundamental mechanisms underlying this age-related memory deficit, we previously developed an integrated, cross-species approach to link converging evidence from human and animal research. This novel approach focuses on the ability to remember sequences of events, an important feature of episodic memory. Unlike existing paradigms, this task is nonspatial, nonverbal, and can be used to isolate different cognitive processes that may be differentially affected in aging. Here, we used this task to make a comprehensive comparison of sequence memory performance between younger (18-22 yr) and older adults (62-86 yr). Specifically, participants viewed repeated sequences of six colored, fractal images and indicated whether each item was presented "in sequence" or "out of sequence." Several out of sequence probe trials were used to provide a detailed assessment of sequence memory, including: (i) repeating an item from earlier in the sequence ("Repeats"; e.g., ABADEF), (ii) skipping ahead in the sequence ("Skips"; e.g., ABDDEF), and (iii) inserting an item from a different sequence into the same ordinal position ("Ordinal Transfers"; e.g., AB3DEF). We found that older adults performed as well as younger controls when tested on well-known and predictable sequences, but were severely impaired when tested using novel sequences. Importantly, overall sequence memory performance in older adults steadily declined with age, a decline not detected with other measures (RAVLT or BPS-O). We further characterized this deficit by showing that performance of older adults was severely impaired on specific probe trials that required detailed knowledge of the sequence (Skips and Ordinal Transfers), and was associated with a shift in their underlying mnemonic representation of the sequences. Collectively, these findings provide unambiguous evidence that the capacity to remember sequences of events is fundamentally affected by typical aging.
\end{abstract}

Episodic memory is generally defined as the memory for personal experiences. In the laboratory, it is typically tested using an "events-in-context" approach, in which subjects are required to remember specific events along with the context in which they occurred (e.g., Yonelinas et al. 2004; Eichenbaum and Fortin 2005; Allen and Fortin 2013). This approach has consistently revealed episodic memory impairments in older adults, characterized by a decrease in the richness of the contextual details associated with specific episodes (Mark and Rugg 1998; Levine et al. 2002; Bastin and Van der Linden 2005; Rajah and D'Esposito 2005). However, the specific nature of such age-associated memory deficits, as well as their progression in older adults, remains poorly understood. This issue is of particular importance because of the need for early detection in the treatment of cognitive disorders, which depend on the ability to distinguish between trajectories associated with typical aging and those linked with pathological changes.

Our understanding of the neuronal mechanisms underlying these progressive age-related memory deficits can be enriched through an integrated, cross-species approach that links converging evidence from human and animal research. For example, the dentate gyrus (DG) is vulnerable to the effects of aging on rodents and primates, with similar age-related vulnerability found in human DG/CA3 regions (West 1993; Gazzaley et al. 1996; Small

\footnotetext{
${ }^{3}$ These authors contributed equally to this work.

Corresponding author: cestark@uci.edu

Article is online at http://www.learnmem.org/cgi/doi/10.1101//m.036301.114.
}

et al. 2002; Penner et al. 2011). In addition, aged rats have reduced field excitatory post-synaptic potentials in the DG (Barnes 1979; Barnes and McNaughton 1980) and LTP-induction deficits at the perforant path-DG synapse (Barnes et al. 2000; Dieguez and Barea-Rodriguez 2004; for review, see Burke and Barnes 2010). Accumulating electrophysiological and behavioral evidence suggest age-related alterations in the DG lead to a reduction in the hippocampus' capacity for pattern separation (Wilson et al. 2006). Inspired by these models, using magnetic resonance imaging, we have identified altered BOLD fMRI responses during a task designed to tax pattern separation in older adults (Yassa et al. 2011a,b) and individuals with amnestic mild cognitive impairment (Yassa et al. 2010). Additionally, we have demonstrated a negative relationship between the degree of alterations of the functional signal (or "representational rigidity") in the DG (combined with CA3 due to resolution limitations) and perforant path integrity in older adults (Yassa et al. 2011a,b), consistent with rodent findings. This translation between rodent and human behavioral neurobiology provides a critical bridge for understanding the underlying physiological and cognitive changes associated with aging and degenerative diseases.

\footnotetext{
C 2015 Allen et al. This article is distributed exclusively by Cold Spring Harbor Laboratory Press for the first 12 months after the full-issue publication date (see http://learnmem.cshlp.org/site/misc/terms.xhtml). After 12 months, it is available under a Creative Commons License (AttributionNonCommercial 4.0 International), as described at http://creativecommons. org/licenses/by-nc/4.0/.
} 
In keeping with the goal of applying rigorous, parallel approaches to further advance our understanding of the neurobiological and cognitive changes in aging, a promising approach is to focus on the ability to remember sequences of events in rodents and humans. Previous research has shown that older adults are impaired at remembering sequences of verbal stimuli (Fabiani and Friedman 1997; Dennis et al. 2003; Bastin and Van der Linden 2005; Giovanello and Schacter 2012) and spatial locations on a screen (Tolentino et al. 2012; Pirogovsky et al. 2013). In addition, lesion studies in rodents (Agster et al. 2002; Fortin et al. 2002; Kesner et al. 2002; Hannesson et al. 2004; DeVito and Eichenbaum 2011) and functional neuroimaging studies in humans (Ekstrom and Bookheimer 2007; Tubridy and Davachi 2011) indicate this capacity depends on the hippocampus and prefrontal cortex, structures known to be specifically disrupted in normal aging, both in rodents and humans (Barnes 1994; Erickson and Barnes 2003; Dennis et al. 2007; Yassa and Stark 2011). Unfortunately, existing sequence paradigms in rodents and humans are not ideally integrated across species in several ways, which precludes thorough comparative and interdisciplinary examinations. First, human studies commonly use verbal stimuli, an approach not available in animal models, and often test cognitive strategies clearly not relevant to the rodent (e.g., creating a short story linking the words). Second, although the use of spatial stimuli offers better crossspecies correspondence, the combination of spatial and temporal demands makes it difficult to study the sequence memory component in isolation, which is particularly problematic since it is well established that the hippocampus plays a critical role in spatial memory (e.g., O'Keefe and Nadel 1978; Burgess et al. 2002).

To address these issues, we used a cross-species sequence memory task we have recently developed (Allen et al. 2014). This task was designed to test the ability of animals and humans to learn and remember sequences of nonspatial and nonverbal items, and illuminate the underlying cognitive processes and mnemonic representations using different types of probe trials. The varying difficulty of these probe trials may serve to uncover memory deficits that can be masked by other, more simple, task designs. In humans, the task involves the repeated presentation of sequences of images (e.g., $\mathrm{ABCDEF}$ ) and requires participants to make a judgment as to whether each item is presented "in sequence" (InSeq) or "out of sequence" (OutSeq). Importantly, Allen et al. (2014) demonstrated that rats and humans exhibit remarkably consistent patterns of performance on this task, thereby providing a critical link with neurobiological research in rodents.

\section{Results}

To directly compare the memory for sequences of events in younger (18-22 yr) and older (62-86 yr) adults, we adapted the crossspecies sequence memory task we previously developed (Fig. 1; Allen et al. 2014). Briefly, participants were tested on two phases of the task: a "no memory" phase which allowed them to become familiar with the basic requirements of the task but did not significantly tax memory, and a "memory" phase which required them to learn and remember new sequences. In each phase, participants received multiple presentations of four distinct sequences on a computer screen and had to determine whether each image was presented "in sequence" (InSeq) or "out of sequence" (OutSeq). Participants were instructed to identify InSeq items by holding a response until the image disappeared, which signaled that the decision threshold (1.0 or $1.2 \mathrm{sec}$ ) had been reached, and OutSeq items by releasing their response as soon as possible (before the decision threshold). While holding a response for correct trials is unusual for human behavioral tasks, we utilized this design to parallel the rodent version of the task in which rats hold in an odor port for correct trials (Allen et al. 2014). To control for the uneven frequency of InSeq and OutSeq items and potential response biases, overall performance was quantified by comparing the "expected" and "predicted" proportions of InSeq and OutSeq responses (see Allen et al. 2014). Based on these proportions, we first calculated $G$-tests (a test similar to the $\chi^{2}$; see Materials and Methods) to determine whether overall performance was significantly better than chance for each participant and phase. Then, to compare performance across groups and phases, we used the same proportions to calculate a Sequence Index (Equation 1; see Materials and Methods), a normally distributed measure that reduces overall performance in each participant to a single number between 0 and 1 . All participants were tested on the two phases of

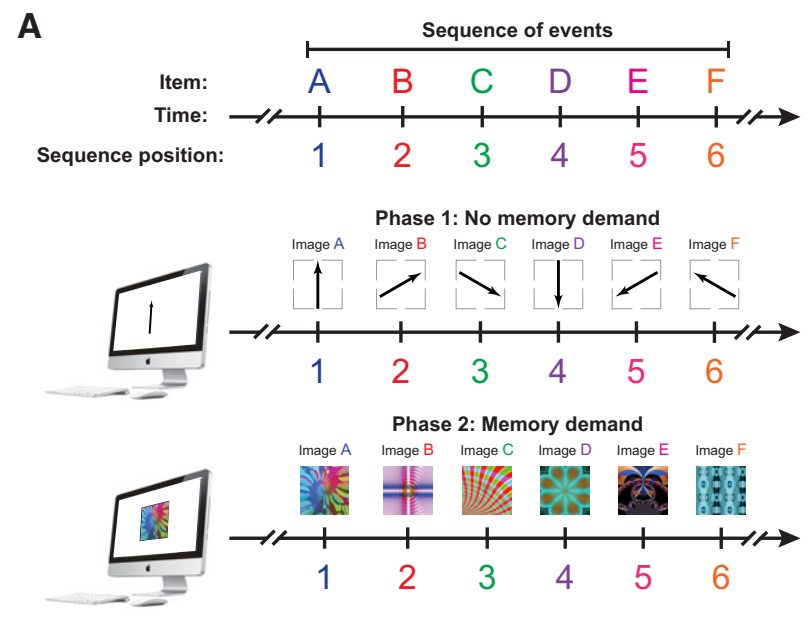

B

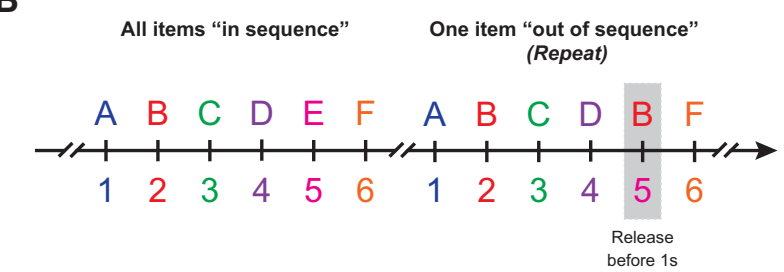

C All items "in sequence" One item "out of sequence"
(Skip)

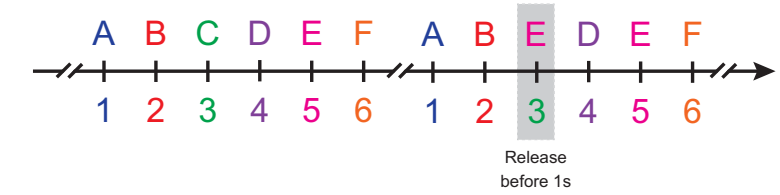

D

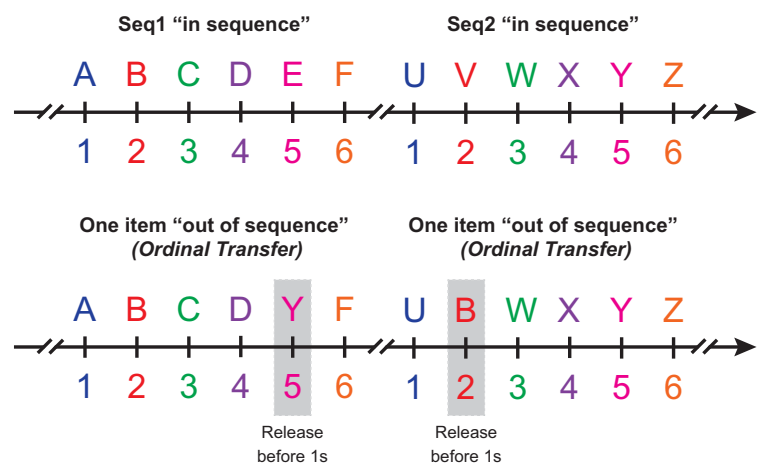

Figure 1. (Legend on next page) 

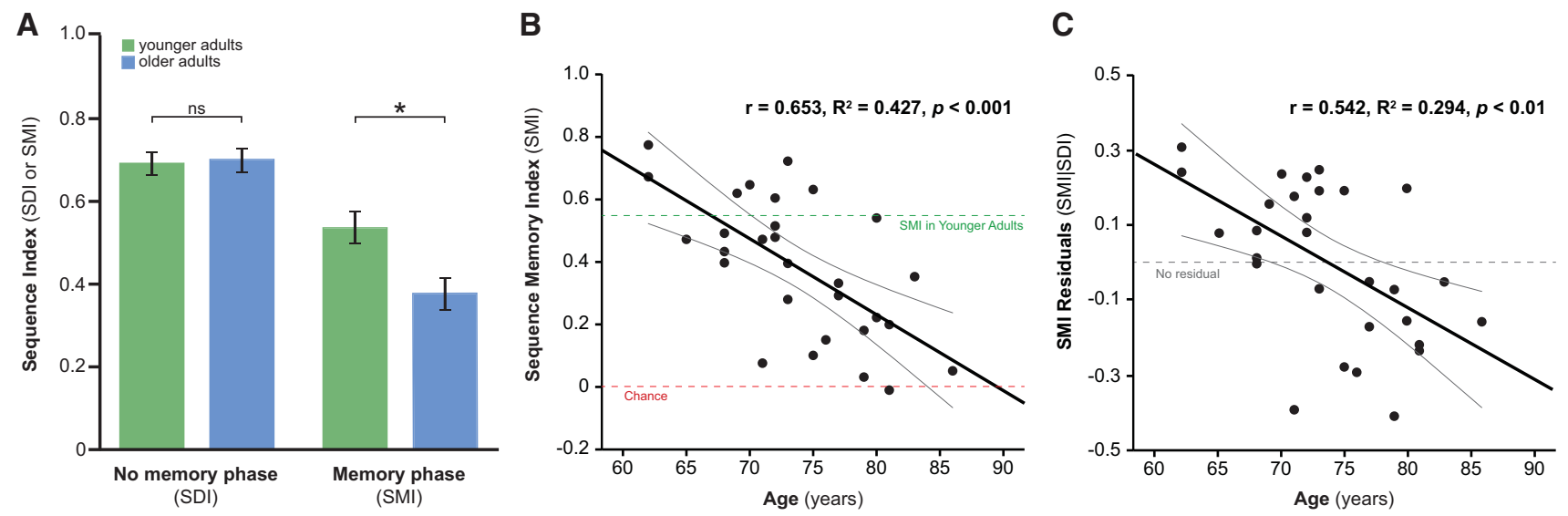

Figure 2. The memory for sequences of events was impaired in older adults. Performance was quantified using a sequence index (Equation 1; Allen et al. 2014), designated sequence detection index (SDI) in the "no memory" phase and sequence memory index (SMI) in the "memory" phase. (A) While younger and older adults performed at a comparably high level in the no memory phase (Age Group main effect: $\left.F_{(1,69)}=0.040, P=0.841\right)$, older adults were significantly impaired in the memory phase $\left(F_{(1,69)}=8.255, P=0.005\right)$. These effects were consistent across the 1.0 and 1.2 sec decision thresholds (combined data shown). Moreover, sequence memory performance (SMI) declined linearly with age in older adults (B) a relationship that remained significant after regressing out the predictive value of SDI scores (SMI|SDI; C), indicating that age-related impairments were not simply due to nonmnemonic performance deficits. Note that the restricted age range in our sample of younger adults precluded a meaningful regression analysis. Regression lines are shown with corresponding 95\% confidence bands. Age Group main effect: $\left(^{*}\right) P<0.05, \mathrm{~ns}, P>0.05$.

the task, with separate groups being tested on the 1.0 or 1.2 sec decision thresholds. Note that we first tested the $1.0 \mathrm{sec}$ threshold, and then added the $1.2 \mathrm{sec}$ to address potential concerns about processing or response speed in the older adults.

\section{Sequence task without memory demand (Phase 1)}

The majority of participants ("Total": 73/77; "Younger": 43/46; "Older": 30/31) demonstrated the ability to detect InSeq and

Figure 1. Behavioral design of the cross-species sequence task (Allen et al. 2014), adapted to compare the memory for sequences of events in younger and older adults. $(A)$ The task was designed to capture the "flow of events" aspect of episodic memory (Tulving 1972, 1984, 2002), which conceptualizes an episode as a sequence of events segmented in time. Participants were tested on two phases of the task: a "no memory" phase in which highly familiar or predictable sequences were presented (e.g., an arrow rotating in clockwise steps), and a "memory" phase in which novel and arbitrary sequences of fractal images were presented. In each phase, participants received multiple presentations of four distinct, interleaved sequences on a computer screen and had to determine whether each image was presented "in sequence" (InSeq) or "out of sequence" (OutSeq). Participants were instructed to identify InSeq items by holding a key until the image disappeared, which signaled that the decision threshold $(1.0$ or $1.2 \mathrm{sec})$ had been reached, and OutSeq items by releasing the key as soon as possible (before the decision threshold). (B-D) Three different types of OutSeq probe trials were used during testing (Repeats, Skips, and Ordinal Transfers), which involve different cognitive processes and sequence representations. Note that probe trials could be presented in any sequence position except the first (i.e., sequences always began with an InSeq item). (B) Repeats occurred when an earlier item was presented a second time in the sequence (e.g., $\mathrm{A} \underline{B} C D B F)$. Repeats can be detected with multiple cognitive strategies and were thus used to define the upper limit of the ability to identify OutSeq items. (C) Skips occurred when an item was presented too early in the sequence (e.g., ABEDEF, which skips over items C and D). Detecting Skips requires accurate predictions of upcoming items and thus performance on these probe trials was used as a sensitive measure of detailed sequence memory. $(D)$ Ordinal Transfers occurred when an item from one sequence (e.g., UVWXYZ) was transferred to another sequence (e.g., $A B C D E F)$ while retaining the item's original ordinal position (e.g., $A B C D Y F)$. Ordinal Transfers were used to help identify the type of sequence representations supporting task performance (i.e., sequential item-item associations or item-in-position associations).
OutSeq items in the "no memory" version of the sequence task ( $G$-tests, $P<0.05)$. The few participants who did not were excluded from the study. No significant differences were found in the frequency of younger and older participants that were able to perform the task in this phase $\left(G_{(1)}=0.433, P=0.511\right)$, indicating that both groups could perform the nonmnemonic aspects of the task.

Performance levels were examined in more detail by calculating the Sequence Index (Equation 1; see Materials and Methods), which in this phase of the task is called the sequence detection index (SDI) because of the lack of any strong memory demand. No significant difference in SDI scores was observed between groups or decision thresholds (Fig. 2A). Specifically, the Age Group $\times$ Decision Threshold ANOVA revealed no main effect of Age Group $\left(F_{(1,69)}=0.040, P=0.841\right)$, Decision Threshold $\left(F_{(1,69)}=\right.$ $0.455, P=0.502)$, and no Age Group $\times$ Decision Threshold interaction effect $\left(F_{(1,69)}=0.304, P=0.583\right)$. These results indicate that both younger and older adults were similar in their ability to detect InSeq and OutSeq items when easily predictable sequences (based on preexisting knowledge) served as stimuli, and thus were capable of executing the performance demands of this sequence task.

\section{Sequence task with memory demand (Phase 2)}

The majority of participants also showed significant memory for novel sequences of fractal images (Total: 67/73; Younger: 41/43; Older: 26/30) and there were no significant differences in the frequency of younger and older adults that showed memory for the sequences $\left(G_{(1)}=1.739, P=0.187\right)$. Note that participants were not excluded from subsequent analyses if they failed to show sequence memory here, as their ability to perform the basic requirements of the task was already established in the "no memory" phase (Phase 1).

Performance levels were examined in more detail with the sequence index (Equation 1; see Materials and Methods), which in this phase is called the sequence memory index (SMI). SMI analyses revealed that older adults were significantly impaired compared with younger adults regardless of decision threshold (Fig. 2A). In fact, the Age Group $\times$ Decision Threshold ANOVA revealed 
Table 1. Mean response times sorted by Age Group, Accuracy, and Decision Threshold for each trial type

\begin{tabular}{|c|c|c|c|c|c|}
\hline \multirow[b]{2}{*}{$\begin{array}{l}\text { Age } \\
\text { group }\end{array}$} & \multirow[b]{2}{*}{ Accuracy } & \multicolumn{2}{|c|}{ InSeq trials } & \multicolumn{2}{|c|}{ OutSeq trials } \\
\hline & & $\begin{array}{c}1.0 \mathrm{sec} \\
\text { decision } \\
\text { threshold }\end{array}$ & $\begin{array}{l}1.2 \mathrm{sec} \\
\text { decision } \\
\text { threshold }\end{array}$ & $\begin{array}{c}1.0 \mathrm{sec} \\
\text { decision } \\
\text { threshold }\end{array}$ & $\begin{array}{c}1.2 \mathrm{sec} \\
\text { decision } \\
\text { threshold }\end{array}$ \\
\hline \multirow[t]{2}{*}{ Younger } & Correct & $1.32 \mathrm{sec}$ & $1.54 \mathrm{sec}$ & $0.69 \mathrm{sec}$ & $0.76 \mathrm{sec}$ \\
\hline & Incorrect & $1.30 \mathrm{sec}$ & $1.54 \mathrm{sec}$ & $0.67 \mathrm{sec}$ & $0.69 \mathrm{sec}$ \\
\hline \multirow[t]{2}{*}{ Older } & Correct & $1.37 \mathrm{sec}$ & $1.54 \mathrm{sec}$ & $0.81 \mathrm{sec}$ & $0.89 \mathrm{sec}$ \\
\hline & Incorrect & $1.35 \mathrm{sec}$ & $1.55 \mathrm{sec}$ & $0.74 \mathrm{sec}$ & $0.89 \mathrm{sec}$ \\
\hline
\end{tabular}

Younger and older adults responded to InSeq and OutSeq trials with similar responses times all conditions.

a significant main effect of Age Group $\left(F_{(1,69)}=8.255, P=0.005\right)$ but no effect of Decision Threshold $\left(F_{(1,69)}=2.157, P=0.147\right)$ and no Age Group $\times$ Decision Threshold interaction effect $\left(F_{(1,69)}=0.701, P=0.405\right)$. As expected, this phase of the task was more difficult than the no memory phase. Both groups showed significantly lower SMI than SDI (Younger: $t_{(42)}=4.556, P<$ 0.001; Older: $\left.t_{(29)}=8.107, P<0.001\right)$, with an effect size twice as large in older adults (Younger: $d=0.696$; Older: $d=1.481$ ).

In order to investigate the relationship between age and performance in the older adult group, a linear regression was conducted using age as a predictor of SMI scores (note that a meaningful regression cannot be performed in younger adults because of the restricted age range). The results show that age was a strong predictor of SMI scores in older adults $\left(r=0.653, R^{2}=0.427\right.$, $\beta=-0.653, F_{(1,27)}=20.087, P<0.001$; Fig. $\left.2 B\right)$. We calculated that the performance of older adults was within the normal range (defined by the $95 \%$ confidence interval, or 2 SD units, of the younger adults group mean) until $71.43 \mathrm{yr}$ of age but was within chance levels (assuming the same confidence interval as above) by $87.6 \mathrm{yr}$ of age. To control for the potential effect of nonmnemonic performance deficits, we performed an additional linear regression using age as a predictor of the SMI residuals that remained after regressing out the predictive value of SDI scores (SMI|SDI; Fig. 2C). Age remained a significant predictor of the SMI residuals $(r=$ $\left.0.542, R^{2}=0.294, \beta=-0.542, F_{(1,27)}=11.221, P<0.01\right)$, suggesting the age-related deficits are specifically due to the demand of remembering sequences of items.

\section{Response times for "in sequence" and "out of sequence" decisions}

It is important to note that InSeq/OutSeq decisions were recorded at approximately the same latencies in older and younger adults: InSeq responses were made $\sim 0.35 \mathrm{sec}$ after the decision threshold and OutSeq responses $\sim 0.2 \mathrm{sec}$ before the decision threshold or earlier (see Table 1). A statistical analysis of response times (Age Group $\times$ Decision Threshold ANOVA) confirmed this observation, revealing a predictable main effect of Decision Threshold $\left(F_{(1,68)}=29.854, P<0.001\right.$; longer response times on longer decision threshold) but no effect of Age Group $\left(F_{(1,68)}=0.466, P=\right.$ $0.497)$ or interaction $\left(F_{(1,68)}=1.776, P<0.187\right)$.

\section{Performance on "out of sequence" probe trials}

While the analysis of SMI scores demonstrated that older adults have significant impairments in the ability to remember sequences, this measure does not shed light on the underlying cognitive processing nor mnemonic representation used by participants. To investigate this issue, we examined the performance of younger and older adults on the three types of OutSeq probe trials: Repeats, Skips, and Ordinal Transfers (see Fig. 1B-D). Since performance on probe trials focused exclusively on OutSeq items, we used accuracy (percent correct) as the dependent measure since the Sequence Index could not be calculated. While accuracy on InSeq items did not differ between younger and older adults $\left(t_{(71)}=-0.116, P=0.908\right)$, the following group differences were observed on OutSeq probe trials.

\section{Repeats}

Older adults were impaired on Repeats (e.g., ABCD吕; Fig. 1B) with a $1.0 \mathrm{sec}$ decision threshold, but there was no such impairment with a $1.2 \mathrm{sec}$ decision threshold (Fig. 3). In fact, the ANOVA revealed a main effect of Age Group $\left(F_{(1,69)}=8.387, P=\right.$ $0.005)$, Decision Threshold $\left(F_{(1,69)}=4.494, P=0.038\right)$, and a significant interaction effect of Age Group $\times$ Decision Threshold $\left(F_{(1,69)}=5.107, P=0.027\right)$.

Performance on Repeats was further examined using Age Group $\times n$-back Distance ANOVAs. For both decision thresholds, performance declined with $n$-back Distance $\left(1.0\right.$ sec: $F_{(3,105)}=$ $4.869, P=0.003 ; 1.2$ sec: $\left.F_{(3,102)}=5.589, P=0.001\right)$ but only in the $1.0 \mathrm{sec}$ data were older adults impaired (main effect of Age
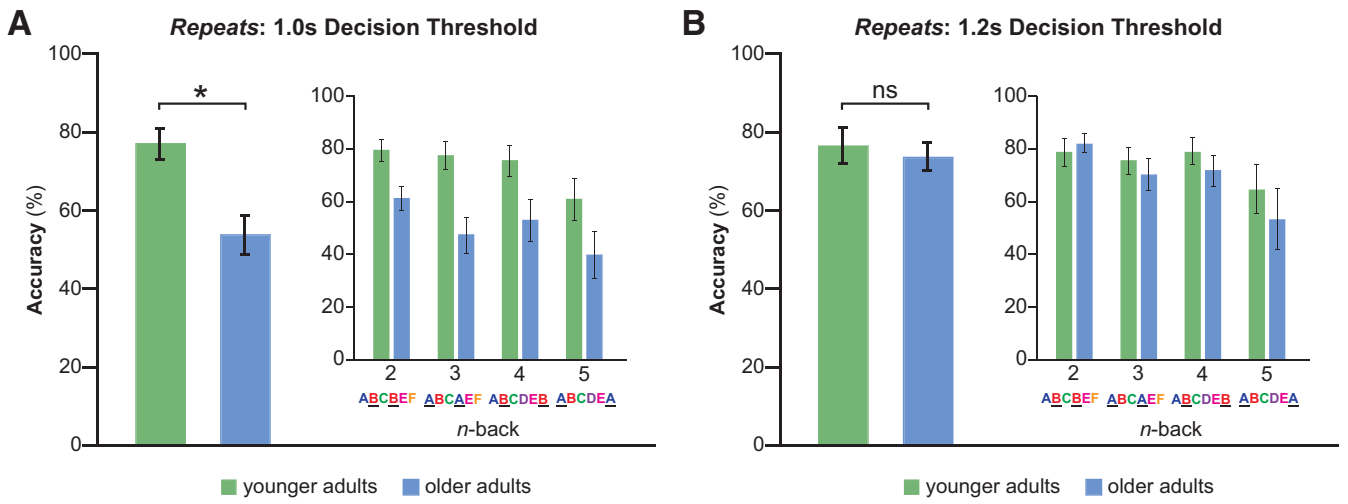

Figure 3. Older adults were impaired on Repeats when tested with a $1.0 \mathrm{sec}$ decision threshold, but performed as well as younger adults with a $1.2 \mathrm{sec}$ decision threshold. (A) Performance on Repeats, probe trials in which an item is a repeat presentation of one that occurred earlier in the sequence, in participants tested with a $1.0 \mathrm{sec}$ decision threshold. The main bar graph shows overall accuracy for younger and older adults, and the inset graph shows the same data sorted by $n$-back distance (how many items back did the item first occur; e.g., ABCBEF is a two-back Repeat). Older adults were significantly impaired in identifying Repeats, a deficit that was consistent across $n$-back distances (Age Group main effect: $F_{(1,35)}=11.72, P=0.002$ ). $(B)$ Corresponding data for participants tested with a $1.2 \mathrm{sec}$ decision threshold. Both younger and older adults performed at a comparably high level, with no significant group differences overall (Age Group main effect: $F_{(1,34)}=0.4844, P=0.491$ ). Age Group main effect: $\left(^{*}\right) P<0.05$, ns, $P>0.05$. 
A

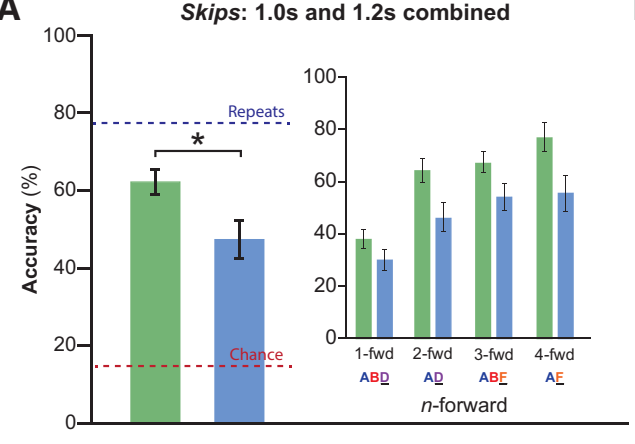

younger adults $\square$ older adults
B

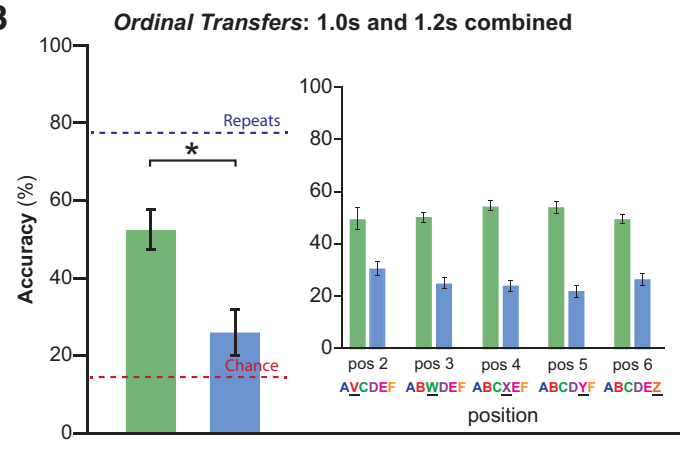

younger adults $\quad$ older adults

Figure 4. Older adults were impaired on Skips and Ordinal Transfers, regardless of the decision threshold. For simplicity, graphs display data combining the two decision thresholds, as the same pattern of results was observed in the 1.0 and $1.2 \mathrm{sec}$ groups (see text). (A) Performance on Skips, probe trials in which the sequence "skips ahead" and items appear too early in the sequence. The main bar graph shows overall accuracy on Skips, and the inset graph shows the same data sorted by $n$-forward distance (the number of items skipped; e.g., ABDDEF is a 1-forward Skip). Older adults exhibited an overall impairment in detecting Skips across $n$-forward distances (Age Group main effect: $F_{(1,69)}=\overline{7.23}, P=0.013$ ), suggesting their memory of the sequences was less detailed thus limiting their ability to predict upcoming items. (B) Performance on Ordinal Transfers, probe trials in which an item is presented in the correct ordinal position but in the wrong sequence (e.g., AVCDEF; main bar graph: overall accuracy, inset graph: same data sorted by ordinal position of transferred item). This type of probe trial was used to help identify the type of mnemonic representations of sequences supporting performance on the task: correctly identifying Ordinal Transfers as OutSeq at asymptotic levels (at the level of Repeats) would be indicative of the use of sequential item-item associations (e.g., since V should not follow A), while accuracy at chance levels would be indicative of item-in-position associations (e.g., failing to detect that $\mathrm{V}$ is OutSeq because its ordinal position is correct). For both older and younger adults, Ordinal Transfers accuracy was significantly below that of Repeats (Older: $t_{(30)}=-13.745, P<0.001$; Younger: $\left.t_{(42)}=-7.419, P<0.001\right)$ and significantly above chance $\left(\right.$ Older: $t_{(30)}=2.361, P=0.025$; Younger: $\left.t_{(42)}=10.654, P<0.001\right)$, suggesting that both groups solved the task using a combination of sequential item-item associations and item-in-position associations. However, older adults identified significantly fewer Ordinal Transfers as OutSeq than younger subjects (Age Group main effect: $\left.F_{(1,69)}=28.160, P<0.001\right)$, suggesting they have a specific decline in memory for sequential item-item associations and thus relied mostly on item-in-position associations to remember sequences. Age Group main effect: $\left(^{*}\right) P<0.05$.

Group; 1.0 sec: $F_{(1,35)}=11.72, P=0.002 ; 1.2$ sec: $F_{(1,34)}=0.484$, $P=0.491)$. Neither decision threshold showed a significant Age Group $\times n$-back Distance interaction effect $\left(1.0\right.$ sec: $F_{(3,105)}=$ $0.421, P=0.739 ; 1.2$ sec: $\left.F_{(3,102)}=0.589, P=0.624\right)$.

\section{Skips}

Our results show that older adults were impaired on Skips (e.g., ABEDEF, Fig. 1C) compared with younger adults, regardless of the decision threshold (Figs. 4A, 5B). Importantly, the ANOVA revealed main effects of Age Group $\left(F_{(1,69)}=7.232, P=0.009\right)$ and Decision Threshold $\left(F_{(1,69)}=7.695, P=0.007\right)$, but there was no Age Group $\times$ Decision Threshold interaction effect $\left(F_{(1,69)}=\right.$ $0.389, P=0.535)$. Because of the lack of a significant interaction effect, we combined the data from both decision thresholds to further examine the performance using an Age Group $\times n$-forward Distance ANOVA. Older adults exhibited an overall impairment across $n$-forward distances (Fig. $4 \mathrm{~A}$, inset), as suggested by the significant main effect of Age Group $\left(F_{(1,34)}=6.853, P=0.013\right)$ and nonsignificant Age Group $\times n$-forward Distance interaction effect $\left(F_{(3,102)}=1.259, P=0.293\right)$. Both groups showed a similar increase in performance as the number of skipped items increased $\left(F_{(3,102)}=28.67, P<0.001\right)$. As expected, both younger and older adults had more difficulty identifying Skips than Repeats (Younger: Repeats-Skips $=-20.3 \pm 1.8 \%, t_{(42)}=-7.330, \quad P<$ 0.001; Older: Repeats - Skips $=-20.6 \pm 1.8 \%, t_{(29)}=-6.448, P<$ 0.001 ; dependent-samples $t$-tests combining 1.0 and 1.2 sec data).

\section{Ordinal transfers}

As mentioned earlier, this type of probe trial consists of images presented in the correct ordinal position but in the wrong sequence (e.g., ABCD $\underline{\mathbf{Y}}$; Fig. 1D), and was used to examine the underlying sequence representations supporting performance in younger and older adults (Allen et al. 2014; see Discussion). If
A
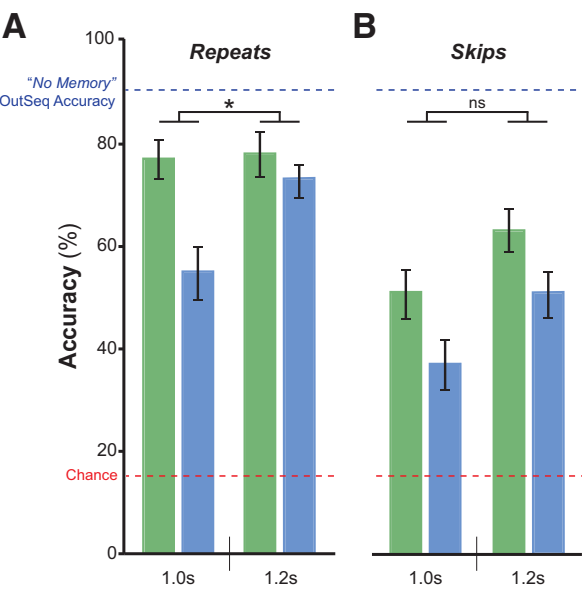

C Ordinal Transfers
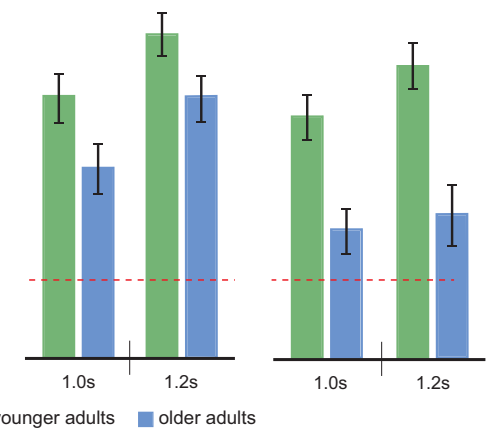

Figure 5. Summary plot showing the effect of decision threshold on Repeats, Skips, and Ordinal Transfers accuracy. Older adults were impaired on Repeats in the $1.0 \mathrm{sec}$ group, but not in the $1.2 \mathrm{sec}$ group (Age Group $\times$ Decision Threshold interaction: $F_{(1,69)}=5.107, P=$ $0.027 ; A)$ While the impairment in the 1.0 sec group may be indicative of a working memory deficit in older adults, the "rescuing" effect of increasing the decision threshold indicates the impairment can be partially attributed to an age-related decline in processing speed. In contrast, impairments of older adults were consistent across decision thresholds for Skips (Age Group $\times$ Decision Threshold interaction: $F_{(1,69)}=0.389, P=$ $0.535 ; B$ ) and Ordinal Transfers (Age Group $\times$ Decision Threshold interaction: $\left.F_{(1,69)}=0.301, P=0.585 ; C\right)$ and thus cannot simply be accounted by a reduction in processing speed. Age Group $\times$ Decision Threshold interaction effect: $\left(^{*}\right) P<0.05 ; \mathrm{ns}, P>0.05$. 
participants relied exclusively on sequential item-item associations to solve the task, then Ordinal Transfers would be identified as OutSeq items (since Y does not follow D) and performance should be at the same level as Repeats (asymptotic performance). Conversely, the same probes would be identified as InSeq items if participants exclusively relied on item-in-position associations (since $\mathrm{Y}$ is in the same ordinal position as $\mathrm{C}$ ) and performance would be at chance levels (accuracy predicted by overall proportion of OutSeq responses). For both older and younger adults, we found that performance on Ordinal Transfers was significantly below Repeats (Older: $t_{(30)}=-13.745, P<0.001$; Younger: $\left.t_{(42)}=-7.419, P<0.001\right)$ and significantly above chance (Older: $t_{(30)}=2.361, P=0.025$; Younger: $\left.t_{(42)}=10.654, P<0.001\right)$, suggesting that the two groups used both types of information to solve the task. However, older adults identified Ordinal Transfers as OutSeq at a significantly lower rate than younger adults across decision thresholds and positions (Figs. 4B, 5C). In fact, the ANOVA revealed a main effect of Age Group $\left(F_{(1,69)}=\right.$ 28.160, $P<0.001)$, but no main effect of Decision Threshold $\left(F_{(1,69)}=1.917, P=0.171\right)$, and no interaction effect of Age Group $\times$ Decision Threshold $\left(F_{(1,69)}=0.301, \quad P=0.585\right)$. As for Skips, because of this nonsignificant interaction effect, performance on Ordinal Transfers was further examined across ordinal positions using data combined from both decision thresholds (Age Group $\times$ Position ANOVA). Similar to Skips, the performance of older adults was significantly lower than younger adults across all positions (Fig. 4B, inset), as indicated by the significant main effect of Age Group $\left(F_{(1,34)}=30.50, P<\right.$ $0.001)$ and nonsignificant interaction effect $\left(F_{(4,136)}=1.654, P=0.164\right)$. However, unlike Skips, performance on Ordinal Transfers did not significantly vary across positions (main effect of Position: $\left.\quad F_{(4,136)}=0.2476, \quad P=0.911\right)$. The finding that older adults identify Ordinal Transfers at a significantly lower rate suggests a shift in the underlying mnemonic representation of the sequences, such as reduction in the use of sequential item-item information or an increase in the use of item-in-position information (see Discussion).

\section{Comparison of sequence memory with RAVLT and BPS-O}

As shown in Figure 2B,C, performance on the sequence memory task (SMI) significantly declined with age in older adults. However, it is unclear how this effect relates to other measures shown to be sensitive to age-related decline over the lifespan, such as the Rey Auditory Verbal Learning Test (RAVLT) (Rey 1941) and the Behavioral Pattern Separation with Objects task (BPS-O) (see Stark et al. 2013 for details). The RAVLT consists of a list of 15 words that are encoded and recalled five times, followed by an interference list, an immediate recall trial, and a delayed recall trial ( $\sim 25$ min later).
The BPS-O compares recognition performance on identical repetitions and on similar lure items. Participants view objects during an indoor/outdoor incidental encoding task, and are then tested in an "old," "similar," and "new" test using repeated items, similar lures, and novel foils. We are particularly interested in performance on the similar lures, in which we have previously reported that accurate "similar" responses decline with age. Participants were all within the normal range for their age on the RAVLT and within the range of their age group on their BPS-O scores (using norms established in Stark et al. 2013).

We ran linear regressions with a subset of the older participants for which we had SMI, RAVLT $(N=17)$ and BPS-O $(N=$ 15) scores. We found no statistically significant relationship between SMI and RAVLT scores ("Immediate RAVLT": $r=0.023$, $R^{2}=0.001, \quad \beta=0.002, \quad F_{(1,15)}=0.008, \quad P=0.932 ; \quad$ "Delayed RAVLT": $r=0.118, R^{2}=0.014, \beta=0.009, F_{(1,15)}=0.219, P=$ 0.646; “Total RAVLT": $\quad r=0.094, \quad R^{2}=0.009, \quad \beta=-0.002$, $F_{(1,15)}=0.137, P=0.717$; Fig. 6 A) nor between SMI and BPS-O scores ("Recognition BPS-O": $r=0.035, R^{2}=0.001, \beta=0.084$,
A

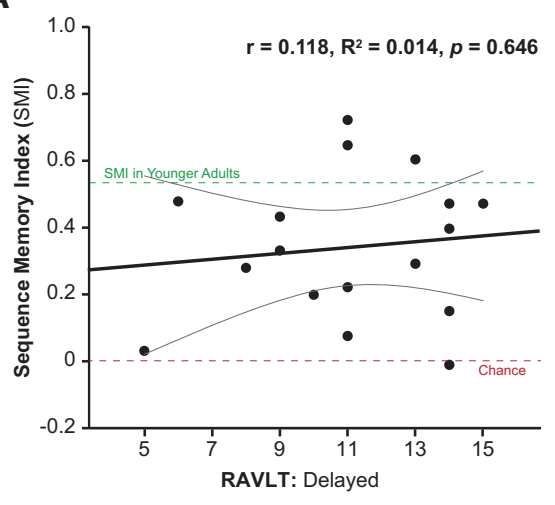

C

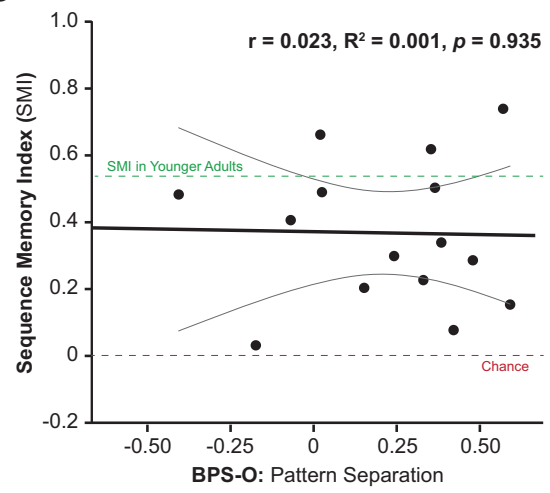

B

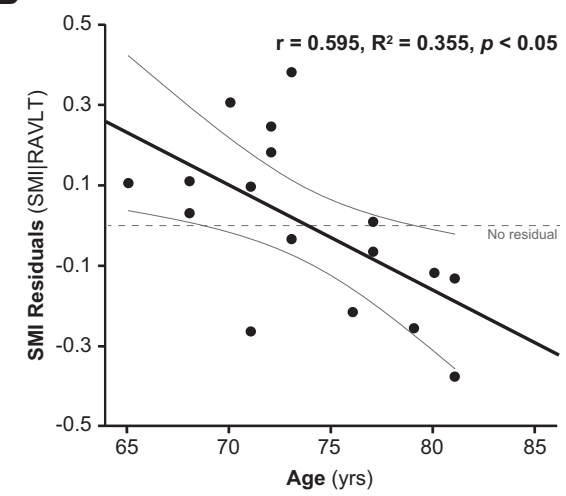

D

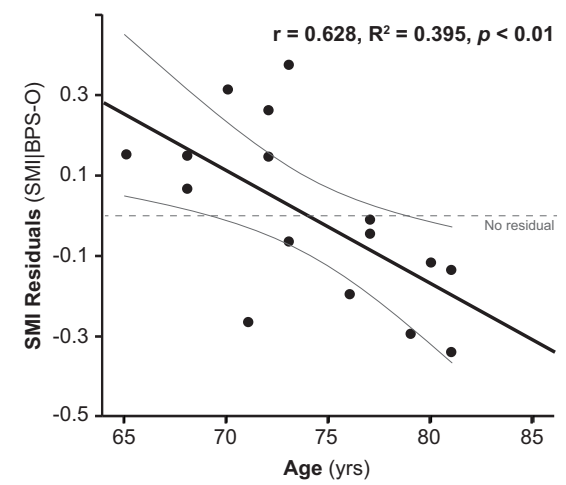

Figure 6. Performance on this novel sequence memory task, measured by SMI scores, provides a sensitive measure to characterize memory decline in aging. Linear regressions were performed to examine the relationship between SMI scores and two measures previously shown to be sensitive to age-related decline over the lifespan (Rey Auditory Verbal Learning Test, RAVLT; Behavioral Pattern Separation with Objects task, BPS-O; see Stark et al. 2013 for details). We found no statistically significant relationship between SMI and RAVLT scores (Immediate, Delayed, or Total scores; Delayed scores shown in A), nor between SMI and BPS-O scores (Recognition or Pattern Separation scores; Pattern Separation scores shown in C). The lack of relationship with SMI scores likely reflects the fact that the age-associated decline of RAVLT and BPS-O scores are typically measured over the lifespan $(\sim 20-85 \mathrm{yr})$ and thus may not be strong predictors within the age range of the older adults group. In contrast, we observed significant relationships between age and SMI residuals after regressing out either the predictive value of RAVLT Delayed Scores (SMI|RAVLT; B) or BPS-O scores (SMI|BPS-O; D), indicating that performance on the sequence memory task captures age-related memory decline not already accounted by RAVLT or BPS-O tests. Regression lines are shown with corresponding $95 \%$ confidence bands. 
$F_{(1,13)}=0.016, P=0.900$; "Pattern Separation BPS-O": $r=0.023$, $R^{2}=0.001, \quad \beta=-0.017, \quad F_{(1,13)}=0.007, \quad P=0.935$; Fig. $\left.6 \mathrm{C}\right)$. Importantly, we found that performance on the sequence memory task, as measured by SMI, captures age-related memory decline not already accounted by RAVLT or BPS-O scores. In fact, we report a significant relationship between age and SMI residuals after regressing out the predictive value of RAVLT Delayed Scores (SMI|RAVLT; $r=0.595, R^{2}=0.355, \beta=-0.596, F_{(1,15)}=8.259$, $\mathrm{P}<0.05$; Fig. $6 \mathrm{~B}$ ) or BPS-O scores (SMI|BPS-O; $r=0.628, R^{2}=$ $0.395, \beta=-0.613, F_{(1,14)}=9.158, P<0.01$; Fig. 6D). Although the sample size is small here, our results suggest that this new sequence memory task may provide a sensitive measure to characterize memory decline in aging that is not being assessed by other memory-based measures. Future studies should address these relationships (or lack thereof) with larger sample sizes to better assess their reliability.

\section{Discussion}

We investigated healthy younger and older adults on their ability to remember sequential relationships among events using a crossspecies sequence task we previously developed (Allen et al. 2014). This task allowed a detailed analysis of sequence memory using three different probe trial types (Repeats, Skips, and Ordinal Transfers; Fig. 1) to examine different cognitive processes and mnemonic representations supporting task performance. The experimental design featured distinct "no memory" and "memory" phases which allowed us to control for any confounding effects of nonmnemonic performance deficits, which may be particularly problematic in older adults. Our results provide compelling evidence that the memory for sequences of events is severely impaired in older adults, that this ability steadily declines with age, and that particular types of sequential processing may be differentially affected by aging.

We began by testing participants in the "no memory" phase of the task (Phase 1), in which we used highly familiar and predictable sequences (e.g., the actual letters "ABCDEF"). We found no significant difference between the performance of younger and older adults (Fig. 2A), indicating that the two groups were matched in their ability to perform the nonmnemonic aspects of the task. Subsequently, participants were tested in the memory phase (Phase 2), in which novel, arbitrary sequences of fractal images were used. As expected, we found that both younger and older adults had more difficulty in the memory phase and, more important, that the decrease in performance was significantly more severe in older adults (Fig. 2A). A linear regression showed that the impairment of older adults represents a graded effect that is predicted by the age of the participants (Fig. 2B), ranging from normal performance at $\sim 70 \mathrm{yr}$ of age to chance levels by $\sim 85 \mathrm{yr}$ of age. To control for the potential influence of nonmnemonic performance deficits, we regressed out the predictive value of the no memory phase and confirmed that the decline in performance with age was due to a loss of memory for new sequences of items (Fig. 2C).

A detailed analysis of performance on probe trials allowed us to further characterize the impairments of older adults. First, we showed that older adults were impaired on Repeats (e.g., $\mathrm{ABCD} \underline{\mathrm{B}}$ ) $)$ at the $1.0 \mathrm{sec}$ decision threshold, but not at the 1.2 sec decision threshold (Figs. 3, 5A). While the impairment on the 1.0 sec condition may reflect the working memory deficits often associated with typical aging (Dobbs and Rule 1989; Baddeley et al. 1999; Logie and Morris 2014), the fact that such deficit is rescued by giving participants more time to respond suggests it could be partially attributed to, or reflect an interaction with, a subthreshold age-related decline in processing speed
(Salthouse 2000). Second, we showed that older adults identified Skips and Ordinal Transfers at a significantly lower rate than younger adults. Importantly, unlike Repeats, these effects were present at both decision thresholds (Fig. 5B,C) and are thus unlikely to be accounted for by a reduction in processing speed. In fact, we chose the $1.2 \mathrm{sec}$ decision threshold because this duration exceeded the vast majority of responses $(>95 \%)$ of older adults on the $1.0 \mathrm{sec}$ condition, thus providing ample processing time.

While the impairment on Skips suggests the memory of the sequences is less detailed in older adults thereby limiting their ability to predict upcoming items, the Ordinal Transfers results suggest age-associated changes in the strategies or representations supporting task performance. Our approach to the examination of underlying strategies was based on the rich history of research on serial order memory in behavioral and cognitive sciences (for reviews, see Lewandowsky and Murdock 1989; Terrace 2005). This literature provides support for the two main mechanisms we discussed: sequential item-item associations (associative chaining models; e.g., Ebbinghaus 1913) and item-in-position associations (positional or ordinal models; e.g., Lashley 1951; Orlov et al. 2000; Terrace 2005). In our paradigm, Ordinal Transfers accuracy at the level of Repeats would be indicative of the exclusive use of sequential item-item associations (e.g., A leads to B, B leads to C, etc.) while accuracy at chance levels would be indicative of the exclusive use of item-in-position associations (e.g., A is first, B is second, etc.). We showed that performance of younger and older adults on Ordinal Transfers was significantly lower than on Repeats but also significantly better than chance, suggesting both age groups relied on the two types of information to solve the task. This view is consistent with recent evidence that models combining elements of associative chaining and temporal context coding can support sequential learning (e.g., pTCM model; Shankar et al. 2009; Howard et al. 2011). Importantly, older adults identified significantly fewer Ordinal Transfers as OutSeq than younger subjects, which may reflect a specific decline in memory for sequential item-item associations and/or an increased reliance on item-in-position associations to remember sequences. It should be noted that the data obtained in this first study cannot fully address predictions from different versions of chaining and temporal context models (e.g., Howard and Kahana 2002; Kahana and Caplan 2002; Polyn and Kahana 2007; Solway et al. 2011) or the potential influence of weaker memories in older adults. To further characterize the mechanisms supporting task performance at the behavioral and neural level, we are currently conducting experiments with a condition in which items from another sequence appear in a different ordinal position (e.g., ABCD2F, where two was in the second ordinal position in a different sequence). Notably manipulations such as these highlight the value of the cross-species sequence task in which sequential elements can be arbitrarily rearranged to probe details of the processes involved in sequence memory.

One challenging aspect of cognitive aging research is that the magnitude of age-related impairments often scales with the task difficulty. Here, for example, no impairment was observed in the "no memory" condition or in the least taxing "memory" condition-the $1.2 \mathrm{sec}$ decision threshold for Repeats. Impairments were observed in the other, more difficult conditions. However, task difficulty alone cannot account for the age-related impairments. For example, in the Skips, a main effect of Decision Threshold was observed, demonstrating that the 1.2 sec condition was easier than the $1.0 \mathrm{sec}$ condition, but there was no evidence for an Age Group $\times$ Decision Threshold interaction (see Fig. 5B). Likewise, there were main effects of Age Group and of $n$-forward Distance (larger distances were less difficult), but no interaction 
for the Skips. A similar main effect of Age Group and $n$-back Distance was observed for the Repeats (larger distances were more difficult), but again no Age Group $\times$ Decision Threshold interaction was observed. Thus, the age-related deficits cannot be accounted for on the basis of difficulty.

Although there is a general consensus that aging results in a decline in episodic memory, the different cognitive processes underlying this capacity may be differentially affected. Previous research has shown age-related deficits for several types of contextual details associated with specific episodes, such as perceptual features (Naveh-Benjamin and Craik 1996; Pilotti et al. 2003), spatial attributes (Denney et al. 1992), relative recency (Fabiani and Friedman 1997; Cabeza et al. 2000), the source of the information (Johnson et al. 1993; Simons et al. 2004) and the computational process of pattern separation (Yassa et al. 2011a,b; Stark et al. 2013). Here we show that aging also results in a severe impairment in the ability to learn and remember specific sequences of items, a capacity critical for capturing the "flow of events" as they occur during a personal episode (Tulving 2002). Importantly, the strong cross-species correspondence of our task (see Allen et al. 2014) provides a unique opportunity to link this work in humans with basic research in rodents.

The structure of this sequence memory task differs from others in the literature in important ways. First, it consists of a series of events presented in the same spatial location. Many paradigms conflate temporal or sequential learning with spatial locations, making independent effects difficult to disentangle (e.g., Madsen and Kesner 1995). For example, Tolentino et al. (2012) and Pirogovsky et al. (2013) developed an elegant design modeled after a rodent 8-arm maze for use in humans to evaluate temporal sequence learning and reported that older adults were impaired relative to young adults on the sequence of locations. Likewise, Rajah et al. (2010a,b) and Duarte et al. (2008) utilized a paradigm that presented images in varying spatial locations and later queried which of two items came earlier than the other or in which spatial location. These studies reported deficits in temporal and spatial context memory in aging, which were also linked to hippocampal volume and changes in prefrontal cortex and hippocampal activity. The data that we report here complement these findings using a paradigm without spatial demands and extend them by providing a particularly rich data set that allows us to examine different error types. In this design, we can evaluate the precise order of items (not simply which came earlier of any given pair of items), the effect of lag or intervening items in the sequence, and the relationship between sequences with Ordinal Transfers. The differences in these error types highlight the different underlying processes and brain regions that may be affected in the course of normal aging. Finally, it should be noted that participants may have used a spatial strategy to remember the sequences (such as tagging each image to a location on a street and then imagining walking down a street, retrieving them in the appropriate order) despite the nonspatial nature of the task. However, none of the participants reported using such a strategy, or any other spatial-type strategy, in our post-testing interviews.

There has been mounting evidence implicating alterations in prefrontal cortex and hippocampal functioning in aging. In humans, the prefrontal cortex shows the steepest rate of age-related atrophy (Pfefferbaum et al. 1998; Raz et al. 2005) and this atrophy has been linked to cognitive decline (Gunning-Dixon and Raz 2003). Similarly, functional neuroimaging studies have shown age-related decreases in prefrontal activity during source memory (Dennis et al. 2008) as well as during encoding and retrieval of relational word pairs (Cabeza et al. 1997; Giovanello and Schacter 2012). Likewise, age-related alterations in functional activity in the hippocampus have been reported for relational memory performance (Tsukiura et al. 2011) and for binding objects to their locations (Mitchell et al. 2000). Finally, alterations in pattern separation performance, stemming from underlying changes in the dentate gyrus subfield of the hippocampus, have also been reported in healthy aging (Yassa et al. 2011a,b).

While our task does not specifically test prospective memory, there are a number of similarities between our task and prospective memory paradigms, which also show deficits in healthy aging (Glisky 1996). Studies of prospective memory often focus on monitoring for a target while engaging in another type of task (Martin et al. 2007). A similar monitoring demand may be required in our task. Prospective memory is thought to involve creating a representation and binding intention to an anticipated event, a function associated with the hippocampal system (McDaniel and Einstein 2007). This information is then fed to the prefrontal cortex, which holds current tasks and intended actions in mind. Correspondingly, different probe trials in our task may differentially tap into hippocampal and prefrontal functioning. The ability to identify Skips and Repeats may depend more heavily on hippocampal binding processes and on prefrontal decision-making processes, respectively. In fact, preliminary data from our laboratory suggest such a dissociation using localized inactivations and single-cell recordings in the hippocampus and prefrontal cortex of rats (Allen et al. 2011, 2013; Quirk et al. 2013). In future studies in rodents and humans, we plan to further evaluate the degree to which these trial types reflect the processing in these regions and how they may be modulated by healthy aging. Note that this sequence task is ideally suited for imaging studies, with preliminary evidence highlighting the role of the hippocampus and medial prefrontal cortex in young adults (Boucquey et al. 2014).

The cross-species sequence memory task has the potential to identify different clinical trajectories in older adults. Interestingly, we did not find a correlation with a common test of dementia (RAVLT; Rey 1941) or with a sensitive test of behavioral pattern separation (BPS-O) (Stark et al. 2013), and thus this task may capture mnemonic processes that are not currently being captured by these other measures. It is possible that different neurobiological factors contribute to memory for sequences of events, such as hippocampal-prefrontal interactions (Quirk et al. 2013), and that these are particularly susceptible to aging; in contrast, RAVLT and BPS-O may be more dependent on entorhinal-hippocampal interactions through the perforant path (Yassa et al. 2011a,b; Yassa and Stark 2011). Adding the crossspecies sequence task to a clinical test battery may improve our ability to identify distinct forms of age-related neurological changes.

Memory for sequences of events may also prove useful in predicting future cognitive decline, such as Alzheimer's disease. Studies show that the risk of dementia of the Alzheimer's type increases dramatically in individuals between the ages of 65 and 85 (Bondi et al. 2008), notably a similar range in which we report age-related sequence memory impairments. There is evidence to suggest that the prevalence of the disease may continue to increase into the ninth decade of life (Jorm and Jolley 1998; von Strauss et al. 1999). This poses a significant concern considering individuals $85+$ yr of age represent the fastest growing population in the country (http://www.alz.org). Therefore, it has become increasingly important to develop methods and to identify markers for differentiating between normal age-related changes and pathological changes. The present study demonstrates that the cross-species sequence task is a sensitive measure of agerelated memory impairments, and may also prove useful as an early detection test for cognitive dysfunction associated with Alzheimer's disease. 
Table 2. Demographical and neuropsychological data on participants

\begin{tabular}{|c|c|c|c|c|}
\hline \multirow{2}{*}{$\begin{array}{l}\text { Age group } \\
\text { Decision threshold }\end{array}$} & \multicolumn{2}{|c|}{ Younger $(n=46)$} & \multicolumn{2}{|c|}{ Older $(n=31)$} \\
\hline & $1.0 \mathrm{sec}$ & $1.2 \mathrm{sec}$ & $1.0 \mathrm{sec}$ & $1.2 \mathrm{sec}$ \\
\hline \multicolumn{5}{|l|}{ Demographics } \\
\hline Age (years)* & $19.83 \pm 1.24$ & $19.73 \pm 1.32$ & $72.31 \pm 6.96$ & $73.93 \pm 6.80$ \\
\hline Education (years)* & $13.79 \pm 1.22$ & $13.09 \pm 3.18$ & $17.25 \pm 2.27$ & $17.07 \pm 1.98$ \\
\hline Sex & $7 \mathrm{M} / 17 \mathrm{~F}$ & $6 \mathrm{M} / 16 \mathrm{~F}$ & $4 \mathrm{M} / 12 \mathrm{~F}$ & $4 \mathrm{M} / 11 \mathrm{~F}$ \\
\hline
\end{tabular}

Data are reported as the mean \pm 1 standard deviation. There were no differences in age or education between the two decision thresholds within each group. *There was a signification difference in age and education between Younger and Older adults, $P>0.05$.

\section{Materials and Methods}

\section{Participants}

This study included 77 participants (46 younger and 31 older adults; see Table 2). There was a significant difference between the two groups in age $\left(t_{(75)}=51.88, \mathrm{P}<0.001\right)$ and years of education $\left(t_{(75)}=7.05, P<0.001\right)$. Older adults were screened for dementia using the mini mental state exam (MMSE) (Folstein et al. 1975). All older adults scored within the normal range on the MMSE (27-30) and thus were considered cognitively intact. Written consent was obtained in compliance with the University of California, Irvine Institutional Review Board (IRB). All participants were compensated for their participation.

\section{Behavioral equipment and stimuli}

Participants were tested in a quiet experimental room on a computer using custom-written MATLAB scripts. Image presentations and response measurements were performed using the Psychophysics Toolbox extensions (http://psychtoolbox.org). Each image presentation $(15 \times 15 \mathrm{~cm}$ on a white background; Fig. 1A) was initiated by pressing and holding down the space bar key. Images disappeared when the spacebar was released, or after the decision threshold ( 1.0 or $1.2 \mathrm{sec}$ ) was reached. The presentation of the next image in the sequence was self-paced, initiated by again pressing and holding down the spacebar key. All six items in a sequence were presented each time, even following an OutSeq trial. The statement "press the spacebar to begin the next sequence" was displayed on screen after each completed sequence to facilitate sequence segmentation. Selected fractal images were highly distinct and abstract, which limited the extent to which they could be described or coded verbally.

\section{“Out of sequence” probe trials}

To help identify the type of cognitive processes, strategies or sequence representations used to support task performance, we included three types of "out of sequence" (OutSeq) probe trials: Repeats, Skips, and Ordinal Transfers (Fig. 1B-D). Probe trials could be presented in any sequence position except the first (i.e., sequences always began with an InSeq item).

\section{Repeats}

OutSeq items included images that were "Repeat" presentations of one that occurred earlier in the sequence (e.g., "ABCD $\underline{\mathbf{B}} \mathrm{F}^{\prime}$ ) (Fig. $1 B)$. Note that Repeats can be detected using multiple cognitive strategies, including sequence memory (e.g., "B should not come after $\mathrm{D}$ ") and working memory (e.g., "B was already presented in this sequence").

\section{Skips}

OutSeq items also included images that "skipped ahead" and thus appeared too early in the sequence (e.g., "ABEDEF"; Fig. 1C). Detecting Skips requires a detailed knowledge of the sequence, necessitating accurate predictions of upcoming items (e.g., "B should be followed by C, not E").

\section{Ordinal transfers}

This type of probe trial was used to help identify the type of mnemonic representations of sequences supporting performance on the task. There are two main types of sequence representations relevant to our paradigm: (i) a directional associative link with the last item (e.g., B leads to C; sequential item-item associations), or (ii) an association between an item and an ordinal position (e.g., B occurs in the second position; item-in-position associations). The degree to which each representation is used can be evaluated by transferring an item from one sequence set (e.g., UVWXYZ) into a second sequence set (e.g., ABCDEF), while retaining the ordinal position (e.g., ABCDYF; Fig. 1D). If participants relied exclusively on sequential itemitem associations to solve the task, then Ordinal Transfers would be identified as OutSeq items (since Y does not follow D). Conversely, the same probes would be identified as InSeq items if participants exclusively relied on item-in-position associations (since $\mathrm{Y}$ is in the same ordinal position as $\mathrm{E}$ ).

\section{Phase 1: no memory demand}

The "no memory" version of the task used sequences of images for which the order of items was easily predictable based on preexisting knowledge (Fig. 1A). Four sequences of six items were used: (1) the actual letters A-F, (2) a clockwise arrow that rotated $60^{\circ}$ each image, (3) bars that moved from left to right and followed the basic colors of the rainbow, and (4) a black dot that moved in equal steps from the upper left to the lower right hand corner of the field of view. Participants initially passively viewed each sequence one time and were then tested on 20 presentations of each sequence (80 sequences in total, randomly interleaved). Half of the sequences had one OutSeq item, which could either be a Repeat or a Skip (20 of each type, randomly selected).

\section{Phase 2: memory demand}

In this phase, novel sequences of fractal images were presented. The fractal images had no intrinsic associations or similarities, which ensured that sequential relationships were novel and arbitrary, thereby placing significant mnemonic demand on participants. Participants were presented with four sequences, each composed of six distinct fractal images. Initially, they passively viewed each of the four sequences once each and were then tested over two stages. The first stage included Repeats and Skips, but no Ordinal Transfers, and consisted of 80 sequence presentations (20 of each sequence, randomly interleaved). Half of the sequences had one OutSeq item, which could either be a Repeat or a Skip (20 of each type, randomly selected). The second stage included all three probe trial types and consisted of a total of 120 sequence presentations (30 per sequence, randomly interleaved). In this stage, two-thirds of the sequences had one OutSeq item, resulting in a total of 20 Repeats, 20 Skips, and 40 Ordinal Transfers. Each stage of testing was followed by a brief interval at which time the subject was prompted to notify the experimenter to continue to the next stage of testing or to notify the experimenter that testing was complete. Task procedures and instructions remained constant across all stages of testing.

\section{Statistics}

The basic requirement of the sequence memory task is to identify InSeq items by holding the response until the decision threshold is reached ( 1.0 or $1.2 \mathrm{sec}$; signaled by the image disappearing from the screen), and OutSeq items by holding for less than the decision threshold. To control for the fact that InSeq trials were more frequent than OutSeq trial, as well as for potential response biases (e.g., increased overall tendency to hold responses), we quantified overall accuracy by comparing the "observed" and 
expected proportions of InSeq and OutSeq responses using $G$-tests (see Allen et al. 2014). The $G$-test is similar to the $\chi^{2}$ test but is more robust for data sets including cells with smaller frequencies (Sokal and Rohlf 1995). To be included in the analyses, each participant had to show significant learning of the sequences in the "no memory" phase, as demonstrated by a significant $G$-test. Significant $G$-tests were followed up with more detailed analyses of sequence memory performance (see below).

To directly compare performance between younger and older adults across decision thresholds and phases of the task, we calculated a sequence index (SI) (Equation 1; see Allen et al. 2014). In essence, the SI normalized the proportion of InSeq and OutSeq items across conditions and reduced sequence memory performance to a single value ranging from -1 to 1 . A score of " 1 " represents perfect sequence performance, in which a participant would have always held for $\geq 1 \mathrm{sec}$ (or $1.2 \mathrm{sec}$ ) for InSeq items, and $<1 \mathrm{sec}$ (or $1.2 \mathrm{sec}$ ) for OutSeq items. A score of " 0 " refers to chance performance, in which a participant would have responded to InSeq and OutSeq items with the same response pattern (e.g., holding until the decision threshold $90 \%$ of the time for both InSeq and OutSeq items). Negative SI scores (worse than chance) were not observed. Note that the Sequence Index is called sequence detection index (SDI) in the "no memory" phase (Phase 1) and sequence memory index (SMI) in the memory phase (Phase 2 ), and that the values for both indices follow a normal distribution. Note that accuracy on OutSeq probe trials was quantified using percent correct, as SMI calculations require both InSeq and OutSeq proportions.

$$
\mathrm{SI}=\frac{\left(0.9 \times \mathrm{IN}_{\text {correct }}\right)\left(0.1 \times \mathrm{OUT}_{\text {correct }}\right)}{\sqrt{\begin{array}{c}
\left(0.9 \times \mathrm{IN}_{\text {correct }}+0.9 \times \mathrm{IN}_{\text {incorrect }}\right)\left(0.1 \times \mathrm{OUT}_{\text {correct }}+0.1\right. \\
\left.\times \mathrm{OUT}_{\text {incorrect }}\right)\left(0.9 \times \mathrm{IN}_{\text {correct }}+0.1 \times \mathrm{OUT}_{\text {incorrect }}\right) \\
\left(0.9 \times \mathrm{IN}_{\text {incorrect }}+0.1 \times \mathrm{OUT}_{\text {correct }}\right)
\end{array}}}
$$

Performance was analyzed using $2 \times 2$ ANOVAs with Age Group (younger and older) and Decision Threshold (1.0 and 1.2 sec) as between-subjects independent variables, followed up by repeated-measures ANOVAs (Age Group $\times$ Distance/Position of probe trial) for each decision threshold. Post hoc paired $t$-tests were used to examine within-subjects changes across conditions. Linear regressions were used to predict sequence memory based on different measures. Tests were considered statistically significant if $P<0.05$.

\section{Acknowledgments}

This research was supported in part by the National Institute on Aging (award NIH R01 AG034613 to C.E.L.S.), the National Science Foundation (CAREER award IOS-1150292 to N.J.F), the Whitehall Foundation (award 2010-05-84 to N.J.F.) and the University of California, Irvine (C.E.L.S. and N.J.F.). We thank Samantha Rutledge and Kristina Le for their assistance with several aspects of the project, and Clare Quirk for useful comments on the manuscript.

\section{References}

Agster KL, Fortin NJ, Eichenbaum H. 2002. The hippocampus and disambiguation of overlapping sequences. J Neurosci 22: 5760-5768.

Allen TA, Fortin NJ. 2013. The evolution of episodic memory. Proc Natl Acad Sci 110(Suppl 2): 10379-10386.

Allen TA, Jacobs N, Feinberg LM, Bharadwaj KR, Wang MX, Fortin NJ. 2011. Prefrontal cortex neurons code for sequences of events. Soc Neurosci Abstr 37: 97-19.

Allen TA, Kraus BJ, McKenzie SA, Hasselmo ME, Eichenbaum HB, Fortin NJ. 2013. Neural representations of sequences of events in the hippocampus parallel behavioral performance. Soc Neurosci Abstr 39: 578.28.

Allen TA, Morris AM, Mattfeld AT, Stark CE, Fortin NJ. 2014. A sequence of events model of episodic memory shows parallels in rats and humans. Hippocampus 24: 1178-1188.
Baddeley A, Cocchini G, Della Salla S, Logie RH, Spinnler H. 1999. Working memory and vigilance: evidence from normal aging and Alzheimer's disease. Brain Cogn 41: 87-108.

Barnes CA. 1979. Memory deficits associated with senescence: a neurophysiological and behavioral study in the rat. J Comp Physiol Psychol 93: 74-104.

Barnes CA. 1994. Normal aging: regionally specific changes in hippocampal synaptic transmission. Trends Neurosci 17: 13-18.

Barnes CA, McNaughton BL. 1980. Physiological compensation for loss of afferent synapses in rat hippocampal granule cells during senescence. J Physiol 309: 473-485.

Barnes CA, Rao G, Houston FP. 2000. LTP induction threshold change in old rats at the perforant path-granule cell synapse. Neurobiol Aging 21: $613-620$.

Bastin C, Van der Linden M. 2005. Memory for temporal context: effects of ageing, encoding instructions, and retrieval strategies. Memory 13: 95-109.

Bondi MW, Jak AJ, Delano-Wood L, Jacobson MW, Delis DC, Salmon DP. 2008. Neuropsychological contributions to the early identification of Alzheimer's disease. Neuropsychol Rev 18: 73-90.

Boucquey VK, Allen TA, Fortin NJ, Stark CEL. 2014. Memory for sequences of events shows bilateral hippocampal and medial prefrontal activity in humans. Soc Neurosci Abstr 40: 646.04.

Burgess N, Maguire EA, O'Keefe J. 2002. The human hippocampus and spatial and episodic memory. Neuron 35: 625-641.

Burke SN, Barnes CA. 2010. Senescent synapses and hippocampal circuit dynamics. Trends Neurosci 33: 153-161.

Cabeza R, Grady CL, Nyberg L, McIntosh AR, Tulving E, Kapur S, Jennings JM, Houle S, Craik FIM. 1997. Age-related differences in neural activity during memory encoding and retrieval: a positron emission tomography study. J Neurosci 17: 391-400.

Cabeza R, Anderson ND, Houle S, Mangels JA, Nyberg L. 2000. Age-related differences in neural activity during item and temporal-order memory retrieval: a positron-emission tomography study. J Cogn Neurosci 12: 197-206.

Denney NW, Dew JR, Khilstrom JF. 1992. An adult developmental study of the encoding of spatial location. Exp Aging Res 18: 25-32.

Dennis NA, Howard JH Jr, Howard DV. 2003. Age deficits in learning sequences of spoken words. J Gerontol B Psychol Sci Soc Sci 58: P224-P227.

Dennis NA, Daselaar A, Cabeza R. 2007. Effects of aging on transient and sustained successful memory encoding activity. Neurobiol Aging 28: $1749-1758$.

Dennis NA, Hayes SM, Prince SE, Madden DJ, Huettel SA, Cabeza R. 2008. Effects of aging on the neural correlates of successful item and source memory encoding. J Exp Psychol Learn Mem Cogn 34: 791-808.

DeVito LM, Eichenbaum H. 2011. Memory for the order of events in specific sequences: contributions of the hippocampus and medial prefrontal cortex. J Neurosci 31: 3169-3175.

Dieguez DJ Jr, Barea-Rodriguez EJ. 2004. Aging impairs the late phase of long-term potentiation at the medial perforant path-CA3 synapse in awake rats. Synapse 52: 53-61.

Dobbs AR, Rule BG. 1989. Adult age differences in working memory. Psychol Aging 4: 500-503.

Duarte A, Henson RN, Graham KS. 2008. The effects of aging on the neural correlates of subjective and objective recollection. Cereb Cortex 18: 2169-2180.

Ebbinghaus H. 1913. Memory: a contribution to experimental psychology. Teachers College, Columbia University, New York.

Eichenbaum H, Fortin NJ. 2005. Bridging the gap between brain and behavior: cognitive and neural mechanisms of episodic memory. J Exp Anal Behav 84: 619-629.

Ekstrom AD, Bookheimer SY. 2007. Spatial and temporal episodic memory retrieval recruit dissociable functional networks in the human brain. Learn Mem 14: 645-654.

Erickson CA, Barnes CA. 2003. The neurobiology of memory changes in normal aging. Exp Gerontol 38: 61-69.

Fabiani M, Friedman D. 1997. Dissociations between memory for temporal order and recognition memory in aging. Neuropsychologia 35: 129-141.

Folstein MF, Folstein SE, McHugh PR. 1975. "Mini-mental state." A practical method for grading the cognitive state of patients for the clinician. J Psychiatr Res 12: 189-198.

Fortin NJ, Agster KL, Eichenbaum HB. 2002. Critical role of the hippocampus in memory for sequences of events. Nat Neurosci $\mathbf{5}$ : $458-462$.

Gazzaley AH, Siegel SJ, Kordower JH, Mufson EJ, Morrison JH. 1996. Circuit-specific alterations of N-methyl-D-aspartate receptor subunit 1 in the dentate gyrus of aged monkeys. Proc Natl Acad Sci 93: $3121-3125$.

Giovanello KS, Schacter DL. 2012. Reduced specificity of hippocampal and posterior ventrolateral prefrontal activity during relational retrieval in normal aging. J Cogn Neurosci 24: 159-170. 
Glisky EL. 1996. Prospective memory and the frontal lobes. In Prospective memory: theory and applications (ed. Brandimonte M, Einstein GO, McDaniel ME), pp. 249-266. Erlbaum, Mahwah, NJ.

Gunning-Dixon FM, Raz N. 2003. Neuroanatomical correlates of selected executive functions in middle-aged and older adults: a prospective MRI study. Neuropsychologia 4: 1929-1941.

Hannesson DK, Howland JG, Phillips AG. 2004. Interaction between perirhinal and medial prefrontal cortex is required for temporal order but not recognition memory for objects in rats. J Neurosci 24: 4596-4604.

Howard MW, Kahana MJ. 2002. A distributed representation of temporal context. J Math Psychol 46: 269-299.

Howard MW, Shankar KH, Jagadisan UKK. 2011. Constructing semantic representations from a gradually-changing representation of temporal context. Top Cogn Sci 3: 48-73.

Johnson MK, Hashtroudi S, Lindsay DS. 1993. Source monitoring. Psychol Bull 114: 3-28.

Jorm AF, Jolley D. 1998. The incidence of dementia: a meta-analysis. Neurology 51: 728-733.

Kahana MJ, Caplan JB. 2002. Associative asymmetry in probed recall of serial lists. Mem Cognit 30: 841-849.

Kesner RP, Gilbert PE, Barua LA. 2002. The role of the hippocampus in memory for the temporal order of a sequence of odors. Behav Neurosci 116: $286-290$.

Lashley KS. 1951. The problem of serial order in behavior. In Cerebral mechanisms in behavior (ed. Jeffress LA), pp. 112-136, John Wyley \& Sons, New York, NY.

Levine B, Svoboda E, Hay JF, Winocur G, Moscovitch M. 2002. Aging and autobiographical memory: dissociating episodic from semantic retrieval. Psychol Aging 17: 677-689.

Lewandowsky S, Murdock BB Jr. 1989. Memory for serial order. Psychol Rev 96: $25-57$.

Logie RH, Morris RG. 2014. Working memory and ageing. Psychology Press, New York, NY.

Madsen J, Kesner RP. 1995. The temporal-distance effect in subjects with dementia of the Alzheimer type. Alzheimer Dis Assoc Disord 9: 94-100.

Mark RE, Rugg MD. 1998. Age effects on brain activity associated with episodic memory retrieval. An electrophysiological study. Brain 121: 861-873.

Martin T, McDaniel MA, Guynn MJ, Houck JM, Woodruff CC, Bish JP, Moses SN, Kicic D, Tesche CD. 2007. Brain regions and their dynamics in prospective memory retrieval: a MEG study. Int J Psychophysiol 64: 247-258.

McDaniel MA, Einstein GO. 2007. Prospective memory: an overview and synthesis of an emerging field. Sage, Thousand Oaks, CA.

Mitchell KJ, Johnson MK, Raye CL, D'Esposito M. 2000. fMRI evidence of age-related hippocampal dysfunction in feature binding in working memory. Brain Res Cogn Brain Res 10: 197-206.

Naveh-Benjamin M, Craig FI. 1996. Effects of perceptual and conceptual processing on memory for words and voice: different patterns for young and old. QJ Exp Psychol A 49: 780-796.

O'Keefe J, Nadel L. 1978. The hippocampus as a cognitive map. Oxford University Press, Oxford.

Orlov T, Yakovlev V, Hochstein S, Zohary E. 2000. Macaque monkeys categorize images by their ordinal number. Nature 404: 77-80.

Penner MR, Roth TL, Chawla MK, Hoang LT, Roth ED, Lubin FD, Sweatt JD, Worley PF, Barnes CA. 2011. Age-related changes in Arc transcription and DNA methylation within the hippocampus. Neurobiol Aging 32: 2198-2210.

Pfefferbaum A, Sullivan EV, Rosenbloom MJ, Mathalon DH, Lim KO. 1998. A controlled study of cortical gray matter and ventricular changes in alcoholic men over a 5-year interval. Arch Gen Psychiatry 55: 905-912.

Pilotti M, Mead ML, Gallo DA. 2003. Implicit and explicit measures of memory for perceptual information in young adults, healthy older adults, and patients with Alzheimer's disease. Exp Aging Res 29: $15-32$.

Pirogovsky E, Holden HM, Jenkins C, Peavy GM, Salmon DP, Galasko DR, Gilbert PE. 2013. Temporal sequence learning in healthy aging and amnestic mild cognitive impairment. Exp Aging Res 39: 371-381.

Polyn SM, Kahana MJ. 2007. Memory search and the neural representation of context. Trends Cogn Sci (Regul. Ed.) 12: 24-30.

Quirk CR, Allen TA, Fortin NJ. 2013. Temporary inactivations of the hippocampus and prefrontal cortex impair memory for sequences of events. Soc Neurosci Abstr 39: 578.04.

Rajah MN, D'Esposito M. 2005. Region-specific changes in prefrontal function with age: a review of PET and fMRI studies on working and episodic memory. Brain 128: 1964-1983.
Rajah MN, Kromas M, Eun Han J, Pruessner JC. 2010a. Group differences in anterior hippocampal volume and in retrieval of spatial and temporal context memory in healthy young versus older adults. Neuropsychologia 48: 4020-4030.

Rajah MN, Languay R, Valiquette L. 2010b. Age-related changes in prefrontal cortex activity are associated with behavioral deficits in both temporal and spatial context memory retrieval in older adults. Cortex 46: $535-549$.

Raz N, Lindenberger U, Rodrigue KM, Kennedy KM, Head D, Williamson A, Dahle C, Gerstorf D, Acker JD. 2005. Regional brain changes in aging healthy adults: general trends, individual differences and modifiers. Cereb Cortex 15: 1676-1689.

Rey A. 1941. L'examen psychologique dans les can d'encephalopathie traumatique. Arch Psychol 28: 286-340.

Salthouse TA. 2000. Aging and measures of processing speed. Biol Psychol 54: $35-54$.

Shankar KH, Jagadisan UKK, Howard MW. 2009. Sequential learning using temporal context. J Math Psychol 53: 474-485.

Simons JS, Dodson CS, Bell D, Schacter DL. 2004. Specific- and partialsource memory: effects of aging. Psychol Aging 19: 689-694.

Small SA, Tsai WY, DeLaPaz R, Mayeux R, Stern Y. 2002. Imaging hippocampal function across the human life span: is memory decline normal or not? Ann Neurol 51: 290-295.

Sokal RR, Rohlf FJ. 1995. Biometry: the principles and practice of statistics in biological research. pp. 724-743. W. H. Freeman and Company, New York.

Solway A, Murdock BB, Kahana MJ. 2011. Positional and temporal clustering in serial order memory. Mem Cognit 40: 177-190.

Stark SM, Yassa MA, Lacy JW, Stark CEL. 2013. A task to assess behavioral pattern separation (BPS) in humans: data from healthy aging and mild cognitive impairment. Neuropsychologia 51: 2442-2449.

Terrace HS. 2005. The simultaneous chain: a new approach to serial learning. Trends Cog Sci 9: 203-210.

Tolentino JC, Pirogovsky E, Luu T, Toner CK, Gilbert PE. 2012. The effect of interference on temporal order memory for random and fixed sequences in nondemented older adults. Learn Mem 19: $251-255$.

Tsukiura T, Sekiguchi A, Yomogida Y, Nakagawa S, Shigemune Y, Kambara T, Akitsuki Y, Taki Y, Kawashima R. 2011. Effects of aging on hippocampal and anterior temporal activations during successful retrieval memory for face-name associations. J Cogn Neurosci 23: 200-213.

Tubridy S, Davachi L. 2011. Medial temporal lobe contributions to episodic sequence encoding. Cereb Cortex 21: 272-280.

Tulving E. 1972. Episodic and semantic memory. In Organization of memory (ed. Tulving E, Donaldson W), p. 381-402. Academic Press Inc., New York.

Tulving E. 1984. Precis of elements of episodic memory. Behav Brain Sci 7: $223-268$.

Tulving E. 2002. Episodic memory: from mind to brain. Annu Rev Psychol 53: $1-25$.

von Strauss E, Viltanen M, De Ronchi D, Winblad B, Fratiglioni L. 1999 Aging and the occurrence of dementia: findings from a population-based cohort with a large sample of nonagenarians. Arch Neurol 56: 587-592.

West MJ. 1993. Regionally specific loss of neurons in the aging human hippocampus. Neurobiol Aging 14: 287-293.

Wilson IA, Gallagher M, Eichenbaum H, Tanila H. 2006. Neurocognitive aging: prior memories hinder new hippocampal encoding. Trends Neurosci 29: 662-670.

Yassa MA, Stark CEL. 2011. Pattern separation in the hippocampus. Trends Neurosci 34: 515-525.

Yassa MA, Stark SM, Bakker A, Albert MS, Gallagher M, Stark CE. 2010. High-resolution structural and functional MRI of hippocampal CA3 and dentate gyrus in patients with amnestic mild cognitive impairment. Neuroimage 51: 1242-1252.

Yassa MA, Lacy JW, Stark SM, Albert MS, Gallagher M, Stark CE. 2011a. Pattern separation deficits associated with increased hippocampal CA3 and dentate gyrus activity in nondemented older adults. Hippocampus 21: $968-979$.

Yassa MA, Mattfeld AT, Stark SM, Stark CE. 2011b. Age-related memory deficits linked to circuit-specific disruptions in the hippocampus. Proc Natl Acad Sci 108: 8873-8878.

Yonelinas AP, Quamme JR, Widaman KF, Kroll EA, Sauvé MJ, Knight RT. 2004. Mild hypoxia disrupts recollection, not familiarity. Cogn Affect Behav Neurosc 4: 393-400.

Received July 1, 2014; accepted in revised form November 26, 2014. 


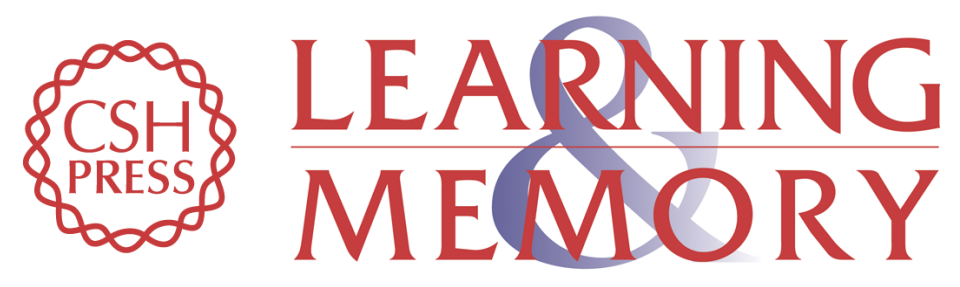

\section{Memory for sequences of events impaired in typical aging}

Timothy A. Allen, Andrea M. Morris, Shauna M. Stark, et al.

Learn. Mem. 2015, 22:

Access the most recent version at doi:10.1101//m.036301.114

References This article cites 80 articles, 9 of which can be accessed free at: http://learnmem.cshlp.org/content/22/3/138.full.html\#ref-list-1

Creative This article is distributed exclusively by Cold Spring Harbor Laboratory Press for the Commons first 12 months after the full-issue publication date (see

License http://learnmem.cshlp.org/site/misc/terms.xhtml). After 12 months, it is available under a Creative Commons License (Attribution-NonCommercial 4.0 International), as described at http://creativecommons.org/licenses/by-nc/4.0/.

Email Alerting Receive free email alerts when new articles cite this article - sign up in the box at the Service top right corner of the article or click here. 